\title{
A REMARK ON CONFIGURATION SPACES OF TWO POINTS
}

\author{
GEORGE RAPTIS AND PAOLO SALVATORE
}

\begin{abstract}
We prove a homotopy invariance result for a certain covering space of the space of ordered configurations of two points in $M \times X$ where $M$ is a closed smooth manifold and $X$ is any fixed aspherical space which is not a point.
\end{abstract}

\section{INTRODUCTION}

It is known that the homotopy type of the ordered configuration space $F_{2}(M)$ of two distinct points in a closed manifold $M$ is not determined by the homotopy type of $M$. Longoni and the second-named author found a counterexample to this homotopy invariance problem in 8 . The counterexample is given by the pair of homotopy equivalent 3-dimensional lens spaces $L_{7,1}$ and $L_{7,2}$. In this case, it turns out that the universal covering spaces of $F_{2}\left(L_{7,1}\right)$ and $F_{2}\left(L_{7,2}\right)$ are also not homotopy equivalent. More pairs of lens spaces have been examined by Evans-Lee [5], providing evidence for the conjecture that any pair of non-homeomorphic lens spaces gives a counterexample. On the other hand, there is a positive result by Levitt [7] who proved the homotopy invariance of $F_{2}(M)$ when $M$ is 2-connected.

The nature of the counterexample suggested the modified question of the homotopy invariance of $F_{2}(M)$ with respect to the simple homotopy type of $M$. This question remains open. An easy way of producing simple-homotopy equivalent manifolds is by taking product with $S^{1}$ : the product property of the Whitehead torsion shows that a homotopy equivalence $f: M \stackrel{\widetilde{\sim}}{\rightarrow} N$ yields a simple-homotopy

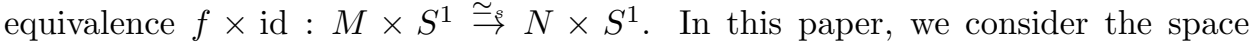
$F_{2}^{S^{1}}\left(M \times S^{1}\right)$ of pairs of points in $M \times \mathbb{R}$ which lie in distinct $\mathbb{Z}$-orbits. This defines a $(\mathbb{Z} \times \mathbb{Z})$-covering space over $F_{2}\left(M \times S^{1}\right)$. A special case of our main result is that the homotopy type of this space is a homotopy invariant of $M$.

More generally, if $X$ is a fixed aspherical space which is not the one-point space, then the homotopy type of a certain covering space of $F_{2}(M \times X)$ is homotopy invariant in $M$ (Theorem 2.5). If $X$ is also contractible, this implies the homotopy invariance of $F_{2}(M \times X)$ (Corollary 2.7). These statements are false, of course, when $F_{2}(X)=\varnothing$, i.e., when $X$ consists of a single point. The proof of Theorem 2.5 uses a description of the covering space of $F_{2}(M \times X)$ as a homotopy pushout (Proposition 2.4) and the fiber homotopy invariance of the spherical tangent bundle of a closed smooth manifold [1.

\section{Configurations of two points in A PRODUCt of SPACES}

Throughout this section, $M$ is a closed smooth manifold and $X$ is a pathconnected Hausdorff space with a basepoint $x \in X$ and a universal covering $p$ : $\widetilde{X} \rightarrow X$. 
2.1. Preliminaries. The configuration space $F_{2}(M) \subset M \times M$ consists of ordered pairs of distinct points in $M$, i.e.,

$$
F_{2}(M)=\left\{\left(m_{1}, m_{2}\right) \in M^{2} \mid m_{1} \neq m_{2}\right\} .
$$

Assume that $M$ has a Riemannian metric $d$. For $\epsilon>0$, we consider the following open subspaces of $M \times M$,

$$
F_{2}(M)_{\epsilon}:=\left\{\left(m_{1}, m_{2}\right) \in F_{2}(M) \mid d\left(m_{1}, m_{2}\right)<\epsilon\right\}
$$

and

$$
D T(M)_{\epsilon}:=\left\{\left(m_{1}, m_{2}\right) \in M \times M \mid d\left(m_{1}, m_{2}\right)<\epsilon\right\} .
$$

There is a (homotopy) pushout square

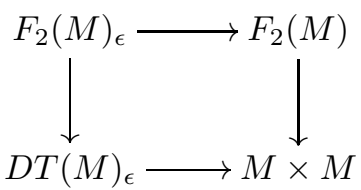

For $\epsilon$ small enough, the projection $F_{2}(M)_{\epsilon} \rightarrow M,\left(m_{1}, m_{2}\right) \mapsto m_{1}$, is homotopy equivalent, fiberwise over $M$, to the spherical tangent bundle of $M$ (see also (7)). The fiber homotopy type of the spherical tangent bundle of $M$ depends only on the homotopy type of $M$ by results of [1, 4]. On the other hand, for $\epsilon$ small, the corresponding projection $D T(M)_{\epsilon} \rightarrow M$ is a homotopy equivalence and fiber homotopy equivalent to the disk tangent bundle of $M$.

2.2. Orbit 2-configurations in a product. Let $G:=\pi_{1}(X, x) \times \pi_{1}(X, x)$. We consider the following covering space of the configuration space of two points in $M \times X$.

Definition 2.1. The $X$-orbit configuration space $F_{2}^{X}(M \times X)$ of two points in $M \times X$ is the covering space of $F_{2}(M \times X)$ defined by

$$
F_{2}^{X}(M \times X):=\left\{\left(\left(m_{1}, x_{1}\right),\left(m_{2}, x_{2}\right)\right) \in F_{2}(M \times \widetilde{X}) \mid\left(m_{1}, p\left(x_{1}\right)\right) \neq\left(m_{2}, p\left(x_{2}\right)\right)\right\} .
$$

The space $F_{2}^{X}(M \times X)$ admits a natural free action by the group $G$ and the quotient is the configuration space $F_{2}(M \times X)$. For $M=*$, the space $F_{2}^{X}(M \times X)$ is the standard orbit configuration space of $\widetilde{X}$, denoted $\widetilde{F}_{2}(X)$. There is a pushout square

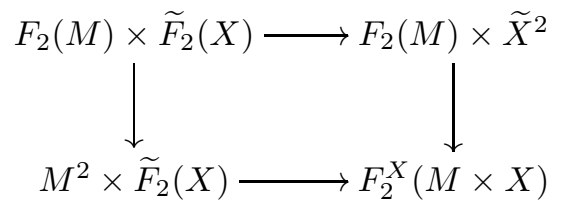

where the maps are the obvious open inclusions. These maps respect the respective actions of $G$ and there is an induced pushout square

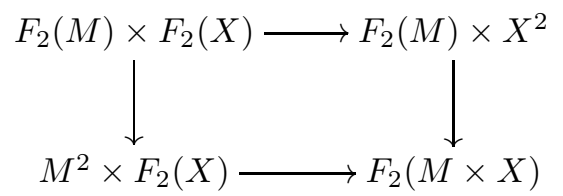

Combining the pushout decompositions in (11) and (2), we obtain the following 
Lemma 2.2. There is a G-equivariant homeomorphism

$$
\left(D T(M)_{\epsilon} \times \widetilde{F}_{2}(X)\right) \bigcup_{F_{2}(M)_{\epsilon} \times \widetilde{F}_{2}(X)}\left(F_{2}(M) \times \widetilde{X}^{2}\right) \stackrel{\cong}{\longrightarrow} F_{2}^{X}(M \times X) .
$$

Proof. This follows easily from the diagram of $G$-equivariant maps

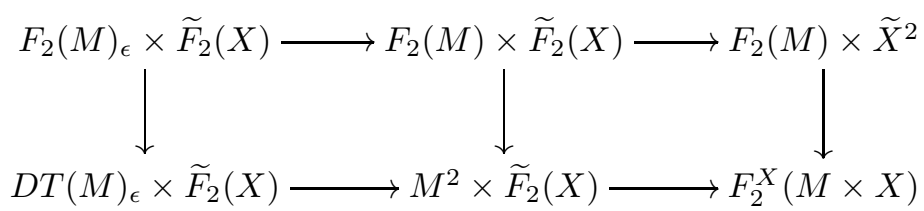

Since both squares are pushouts, so is also the composite square.

Corollary 2.3. Assume that $F_{2}(X)$ is non-empty (i.e., $X$ has at least two points). Then there is a pushout of $G$-equivariant maps

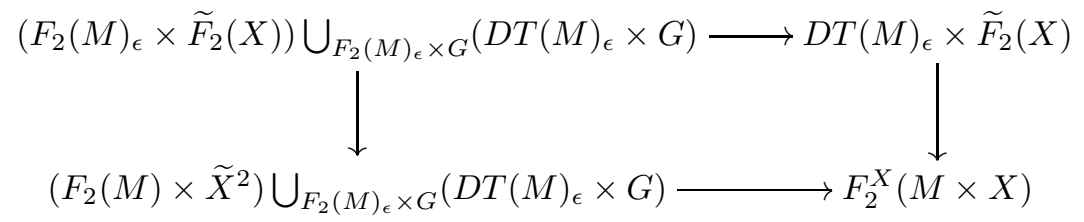

Proof. Let $q: G \rightarrow \widetilde{F}_{2}(X)$ be the inclusion of an orbit where $G$ is regarded as a discrete topological group. Consider the following diagram:

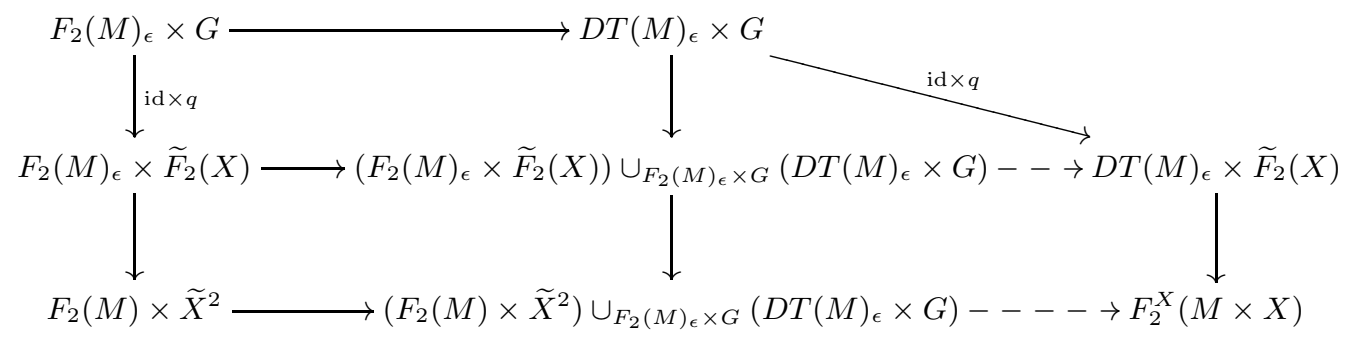

Note that all of the maps respect the corresponding $G$-actions. The squares on the left are pushouts by definition. The composite bottom square is a pushout by Lemma 2.2. Therefore the bottom right square is also a pushout, as required.

2.3. Homotopy Invariance. The somewhat complicated diagram in Corollary 2.3 can be simplified at the expense of losing $G$-equivariance. First, let $\epsilon>0$ be small enough so that the closed inclusion of the subspace of $F_{2}(M)_{\epsilon}$ which consists of those pairs of points which are exactly $(\epsilon / 2)$-apart,

$$
S T(M):=\left\{\left(m_{1}, m_{2}\right) \in M \times M \mid d\left(m_{1}, m_{2}\right)=\epsilon / 2\right\} \stackrel{j}{\hookrightarrow} F_{2}(M)_{\epsilon},
$$

is a homotopy equivalence, the projection $S T(M) \rightarrow M,\left(m_{1}, m_{2}\right) \mapsto m_{1}$, is fiber homotopy equivalent to the spherical tangent bundle of $M$, and the projection $D T(M)_{\epsilon} \rightarrow M$ is a homotopy equivalence. We denote $D T(M):=D T(M)_{\epsilon}$.

We obtain the following homotopy pushout decomposition of $F_{2}^{X}(M \times X)$. Here homotopy pushout is always considered with respect to the weak homotopy equivalences. 
Proposition 2.4. Suppose that $\tilde{X}$ is weakly contractible and $F_{2}(X) \neq \varnothing$ (i.e, $X$ has at least two points). Let $q: * \rightarrow \widetilde{F}_{2}(X)$ be the inclusion of a point. Then the space $F_{2}^{X}(M \times X)$ is weakly equivalent to the homotopy pushout of the maps

$$
M^{2} \longleftarrow\left(S T(M) \times \widetilde{F}_{2}(X)\right) \bigcup_{S T(M) \times\{*\}} D T(M) \times\{*\} \longrightarrow D T(M) \times \widetilde{F}_{2}(X)
$$

which are defined by the projection away from $\widetilde{F}_{2}(X)$ and the point $q$.

Proof. The proof is similar to that of Corollary 2.3. Consider the following commutative diagram

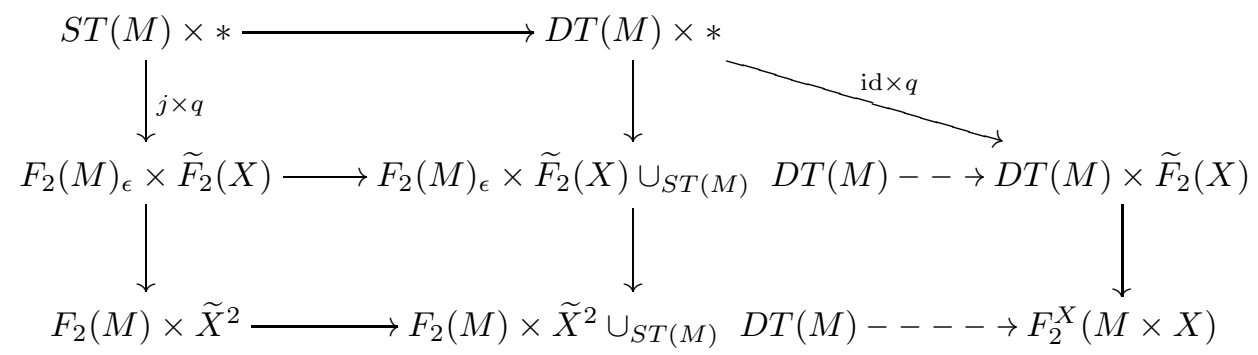

The two squares on the left are pushouts by definition. The top map is a cofibration, therefore they are also homotopy pushouts (see, e.g., 2, Appendix, Proposition 4.8]). The bottom composite square is a pushout by Lemma 2.2. This pushout decomposition of $F_{2}^{X}(M \times X)$ arises from an open covering defined by two open subsets and therefore it defines a homotopy pushout (see also [3] for more general results). It follows that the bottom right square is also a homotopy pushout.

There is an obvious commutative diagram

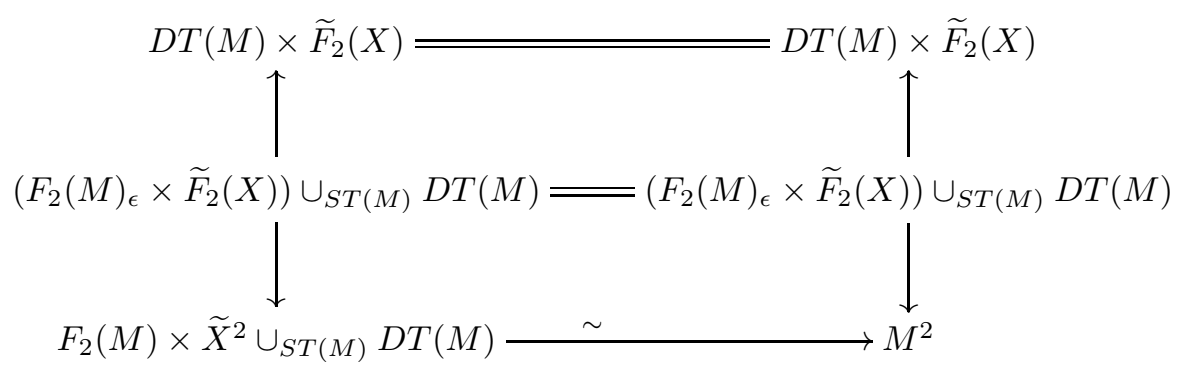

where the bottom map is a weak homotopy equivalence using that $\widetilde{X} \rightarrow *$ is a weak homotopy equivalence, $S T(M) \stackrel{j}{\simeq} F_{2}(M)_{\epsilon}$ is a homotopy equivalence, and the homotopy pushout in Diagram (11). Therefore the homotopy pushouts of the vertical pairs of maps are weakly homotopy equivalent. Similarly, they can be identified with the required homotopy pushout using the homotopy equivalence $S T(M) \stackrel{j}{\simeq} F_{2}(M)_{\epsilon}$ and the fact that $S T(M) \subset D T(M)$ is a cofibration.

Theorem 2.5. Suppose that $X$ has a weakly contractible universal covering space and $F_{2}(X) \neq \varnothing$ (i.e., $X$ has at least two points). If $M$ and $N$ are homotopy equivalent closed smooth manifolds, then the spaces $F_{2}^{X}(M \times X)$ and $F_{2}^{X}(N \times X)$ are weakly homotopy equivalent. 
Proof. By the fiber homotopy invariance of the spherical tangent bundle [1, 4, there is a homotopy commutative square

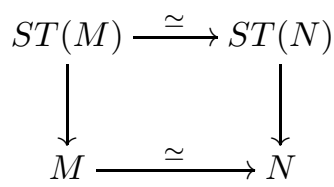

where the vertical maps are (any of) the (two homotopic) projections and the horizontal maps are homotopy equivalences. The projection $D T(M) \rightarrow M$ is a homotopy equivalence and, under this identification, the inclusion $D T(M) \subset M \times$ $M$ is homotopic to the diagonal inclusion $\Delta: M \rightarrow M \times M$. Thus, the homotopy pushout decomposition in Proposition 2.4 is weakly homotopy invariant in $M$ and the result follows.

Corollary 2.6. Let $X, M$ and $N$ be as in Theorem 2.5. Suppose that $\pi_{1}(X)$ is finite. Then there is a zig-zag of maps connecting $F_{2}(M \times X)$ and $F_{2}(N \times X)$ and inducing isomorphisms in rational homology.

Proof. The claim is obvious when $M$ and $N$ are 0-dimensional. If the dimension is positive, the zig-zag of maps is as follows

$$
F_{2}(M \times X) \leftarrow F_{2}^{X}(M \times X) \simeq_{w} F_{2}^{X}(N \times X) \rightarrow F_{2}(N \times X)
$$

where in the middle is the weak homotopy equivalence from Theorem 2.5 and the other two maps are the natural projections. These two maps are finite covering maps and it is easy to check that they induce bijections on $\pi_{0}$. Therefore they induce isomorphisms between the rational homology groups.

Corollary 2.7. Let $M$ and $N$ be homotopy equivalent closed smooth manifolds.

(a) Suppose that $X$ is weakly contractible and $F_{2}(X) \neq \varnothing$. Then $F_{2}(M \times X)$ and $F_{2}(N \times X)$ are weakly homotopy equivalent.

(b) $F_{2}^{S^{1}}\left(M \times S^{1}\right)$ and $F_{2}^{S^{1}}\left(N \times S^{1}\right)$ are homotopy equivalent.

Corollary 2.8. The spaces $F_{2}^{S^{1}}\left(L_{7,1} \times S^{1}\right)$ and $F_{2}^{S^{1}}\left(L_{7,2} \times S^{1}\right)$ are homotopy equivalent.

Since $L_{7,1}$ and $L_{7,2}$ are not homeomorphic, the spaces $L_{7,1} \times S^{1}$ and $L_{7,2} \times S^{1}$ are also not homeomorphic by results of [6] (see, e.g., the proof in [6, p. 177]). However, they are simple-homotopy equivalent because the Whitehead torsion of $f \times \operatorname{id}_{S^{1}}$ vanishes for every homotopy equivalence $f$. In [8], it was shown that the orbit configuration spaces $\widetilde{F}_{2}\left(L_{7,1}\right)$ and $\widetilde{F}_{2}\left(L_{7,2}\right)$ are not homotopy equivalent, thus disproving the homotopy invariance of configuration spaces. It remains open whether the configuration spaces $F_{2}\left(L_{7,1} \times S^{1}\right)$ and $F_{2}\left(L_{7,2} \times S^{1}\right)$ are homotopy equivalent and whether, more generally, the correspondence $M \mapsto F_{2}\left(M \times S^{1}\right)$ is homotopy invariant. Based on the properties of the Whitehead torsion, this problem relates to the general question about the homotopy invariance of configuration spaces with respect to simple-homotopy equivalences.

\section{REFERENCES}

[1] Benlian, René; Wagoner, John, Type d'homotopie fibré et réduction structurale des fibrés vectoriels, C. R. Acad. Sci. Paris Sér. A-B 265 (1967) A207-A209. 
[2] Boardman, J. M.; Vogt, R. M., Homotopy invariant algebraic structures on topological spaces, Lecture Notes in Mathematics, Vol. 347. Springer-Verlag, Berlin-New York, 1973.

[3] Dugger, Daniel; Isaksen, Daniel C., Topological hypercovers and $\mathbb{A}^{1}$-realizations, Math. Z. 246 (2004), no. 4, 667-689.

[4] Dupont, Johan L., On homotopy invariance of the tangent bundle. I, II, Math. Scand. 26 (1970), 5-13; ibid. 26 (1970) 200-220.

[5] Evans-Lee, Kyle, On configuration spaces of lens spaces, Ph.D. Thesis, University of Miami, 2015.

[6] Kwasik, Sławomir; Rosicki, Witold, On stability of 3-manifolds, Fund. Math. 182 (2004), no. 2, 169-180.

[7] Levitt, Norman, Spaces of arcs and configuration spaces of manifolds, Topology 34 (1995), no. $1,217-230$.

[8] Longoni, Riccardo; Salvatore, Paolo, Configuration spaces are not homotopy invariant, Topology 44 (2005), no. 2, 375-380.

G. RAPTIS

Universität Regensburg, Fakultät für Mathematik

93040 Regensburg, Germany

E-mail address: georgios.raptis@ur.de

P. SAlvatore

Dipartimento di Matematica, Università di Roma Tor Vergata

Via della Ricerca Scientifica, 00133 Roma, Italy

E-mail address: salvator@mat.uniroma2.it 